\title{
Optical Absorption by Interlayer Density Excitations in Bilayer Graphene
}

\author{
Tsuneya ANDO and Mikito KOSHINO \\ Department of Physics, Tokyo Institute of Technology \\ 2-12-1 Ookayama, Meguro-ku, Tokyo 152-8551
}

\begin{abstract}
The optical absorption spectrum for light with electric field perpendicular to a bilayer graphene is shown to be quite different from that for parallel polarization. This arises partly from the difference in the selection rule and from the important depolarization effect due to induced polarization. The depolarization effect is most prominent for the sharp transition between the lowest to the first excited conduction band, which are almost parallel to each other.
\end{abstract}

Keywords: graphene, bilayer, two-dimensional graphite, optical transition, depolarization effect, dynamical screening

\section{$\S 1$. Introduction}

Bilayer graphenes consisting of two layers were recently fabricated $^{1)}$ and various phenomena started to be investigated. $^{2-6)}$ The purpose of this paper is to study optical absorption for light with electric field perpendicular to the layer and to clarify the difference from that for light incident normal to the layer.

In an effective-mass approximation, an electron in a monolayer graphene is described by Weyl's equation for a massless neutrino. ${ }^{7-14)}$ Transport properties in such an exotic system are intriguing and the conductivity with/without magnetic field including the Hall effect, ${ }^{15,16)}$ the dynamical transport, ${ }^{17)}$ and quantum corrections to the conductivity ${ }^{18)}$ were theoretically investigated. The system was shown to exhibit various properties different from conventional two-dimensional systems. ${ }^{19)}$

A bilayer graphene has electronic states quite different from those in the monolayer system as was studied for the band structure with or without a magnetic field, ${ }^{20-24)}$ the transport ${ }^{25-29)}$ and dynamical properties, ${ }^{30-32)}$ and diamagnetic susceptibility. ${ }^{32-34)}$ One important feature is that the band structure can be strongly modified due to opening-up of a gap by applied electric field. ${ }^{35-37)}$

The paper is organized as follows: In $\S 2$, the dynamical conductivity describing optical transitions is introduced and some of its properties are discussed for a symmetric bilayer. Explicit numerical results in bilayers with asymmetric potential self-consistently determined are presented in $\S 3$. Results are discussed and a short summary is presented in $\S 4$.

\section{$\S 2$. Formulation}

\subsection{Effective-mass description}

We consider a bilayer graphene which is arranged in the AB (Bernal) stacking. The upper layer is denoted as 1 and the lower layer denoted as 2. In a monolayer graphene the conduction and valence bands consisting of $\pi$ orbitals cross at $\mathrm{K}$ and $\mathrm{K}$ ' points of the Brillouin zone, where the Fermi level is located. ${ }^{38,39)}$ Electronic states near a K point are described by the $\boldsymbol{k} \cdot \boldsymbol{p}$ equation equivalent to Weyl's equation or a Dirac equation with vanishing rest mass. ${ }^{7-14)}$ For the inter-layer coupling, we include only the coupling between vertically neighboring atoms. Then, electronic states are described by the $\boldsymbol{k} \cdot \boldsymbol{p}$ equation: ${ }^{20,25,37)}$

$$
\mathcal{H}_{0} \boldsymbol{F}(\boldsymbol{r})=\varepsilon \boldsymbol{F}(\boldsymbol{r})
$$

with

$$
\mathcal{H}_{0}=\left(\begin{array}{cccc}
e F d / 2 & \gamma \hat{k}_{-} & 0 & 0 \\
\gamma \hat{k}_{+} & e F d / 2 & \Delta & 0 \\
0 & \Delta & -e F d / 2 & \gamma \hat{k}_{-} \\
0 & 0 & \gamma \hat{k}_{+} & -e F d / 2
\end{array}\right)
$$

and

$$
\hat{k}_{ \pm}=\hat{k}_{x} \pm \mathrm{i} \hat{k}_{y}
$$

where $\gamma$ is a band parameter, $\hat{\boldsymbol{k}}=\left(\hat{k}_{x}, \hat{k}_{y}\right)=-\mathrm{i} \boldsymbol{\nabla}$ is a wave-vector operator, $\Delta$ represents the inter-layer coupling, and $e F d$ represents the potential difference between layers 1 and 2 ( $F$ is the effective electric field and $d=3.34 \AA$ is the interlayer distance), as illustrated in Fig. 1 (a).

Parameters $\gamma$ and $\Delta$ are related to tight-binding parameters $\gamma_{0}$ and $\gamma_{1}$ through $\gamma=(\sqrt{3} / 2) a \gamma_{0}$ and $\Delta=\gamma_{1}$, where $a$ is the lattice constant given by $a=2.46 \AA, \gamma_{0} \approx 3$ $\mathrm{eV},{ }^{40)}$ and $\gamma_{1} \approx 0.4 \mathrm{eV}{ }^{41)}$ We shall completely neglect other interlayer couplings because they do not play important roles as has been discussed previously. ${ }^{25,42)}$

The states are specified by the set of quantum numbers $j$ and $\boldsymbol{k}$, with $\boldsymbol{k}$ the wave vector and bands $j=1,2,3,4$ in the order of increasing energy $(j=1$ and 2 for the valence bands and $j=3$ and 4 for the conduction bands). The energy dispersion for varying values of the potential difference $e F d$ is shown in Fig. 2 .

We consider the situation that electron concentration $n_{\mathrm{s}}$ is varied by the bottom gate with a fixed value of the top-gate voltage giving external field $F_{\text {ext }}$. The asymmetry between two layers causes difference in the electron density and then leads to that in the electrostatic potential. Therefore, eFd should be self-consistently determined for each electron concentration. ${ }^{35)}$ Some examples of such calculations were carried out in ref. 37 for the static dielectric constant of the environment, $\kappa=2$. We shall use this result for explicit calculations to be 
presented in the following.

\subsection{Optical Absorption for Perpendicular Polarization}

We apply external electric field $E_{\text {ext }}(\omega) \mathrm{e}^{-\mathrm{i} \omega t}+$ c.c. perpendicular to the layer, where 'c.c.' stands for complex conjugate. Let $n_{1}$ and $n_{2}$ be the electron density in layers 1 and 2, respectively, per unit area. The asymmetry in the density distribution $\Delta n(\omega)$ is defined as

$$
\Delta n(\omega)=n_{2}(\omega)-n_{1}(\omega) .
$$

This induces electric field

$$
(-e) E_{\text {ind }}(\omega)=-\frac{4 \pi e^{2}}{\kappa} \frac{\Delta n(\omega)}{2},
$$

in the region $-d / 2<z<+d / 2$, where $\kappa$ is the static dielectric constant of the environment. This is illustrated in Fig. 1 (b).

We shall define the polarizability $\alpha(\omega)$ through

$$
(-e) \frac{\Delta n(\omega)}{2}=\alpha(\omega) E_{\text {tot }}(\omega),
$$

where $E_{\text {tot }}(\omega) \mathrm{e}^{-\mathrm{i} \omega t}+$ c.c. is the total electric field. Then, we have

$$
\begin{aligned}
(-e) E_{\mathrm{tot}}(\omega) & =(-e) E_{\mathrm{ext}}(\omega)+(-e) E_{\mathrm{ind}}(\omega) \\
& =(-e) E_{\mathrm{ext}}(\omega)-\frac{4 \pi e^{2}}{\kappa} \frac{\Delta n(\omega)}{2},
\end{aligned}
$$

giving

$$
E_{\mathrm{tot}}(\omega)=\frac{E_{\mathrm{ext}}(\omega)}{\varepsilon(\omega)}
$$

with

$$
\varepsilon(\omega)=1+\frac{4 \pi}{\kappa} \alpha(\omega) .
$$

With the use of the equation of continuity, we have induced current

$$
j(\omega)=-\mathrm{i} \omega(-e) \frac{\Delta n(\omega)}{2}=\frac{1}{d} \sigma_{z z}(\omega) E_{\mathrm{tot}}(\omega),
$$

in the region $-d / 2<z<+d / 2$, with two-dimensional conductivity

$$
\sigma_{z z}(\omega)=-\mathrm{i} \omega \alpha(\omega) d
$$

Then, we have

$$
\varepsilon(\omega)=1+\frac{4 \pi \mathrm{i}}{\omega \kappa d} \sigma_{z z}(\omega)
$$

and

$$
j(\omega)=\frac{1}{d} \tilde{\sigma}_{z z}(\omega) E_{\text {ext }}(\omega),
$$

with

$$
\tilde{\sigma}_{z z}(\omega)=\frac{\sigma_{z z}(\omega)}{\varepsilon(\omega)} .
$$

The power absorption per unit area is given by

$$
P=\frac{d}{2} \operatorname{Re}\left[j(\omega) E_{\mathrm{tot}}(\omega)^{*}\right]=\frac{1}{2} \operatorname{Re} \tilde{\sigma}_{z z}(\omega)\left|E_{\text {ext }}(\omega)\right|^{2} .
$$

The dynamical conductivity can easily be calculated in a linear response theory. Define

$$
\hat{\tau}=\left(\begin{array}{cccc}
+1 & 0 & 0 & 0 \\
0 & +1 & 0 & 0 \\
0 & 0 & -1 & 0 \\
0 & 0 & 0 & -1
\end{array}\right) .
$$

Then, we have

$$
\sigma_{z z}(\omega)=\mathrm{i} \omega e^{2} d^{2} \frac{g_{\mathrm{v}} g_{\mathrm{s}}}{4} \frac{1}{L^{2}} \sum_{\alpha, \beta} \frac{\left(f_{\alpha}-f_{\beta}\right)\left|\hat{\tau}_{\alpha \beta}\right|^{2}}{\varepsilon_{\alpha}-\varepsilon_{\beta}+\hbar \omega+\mathrm{i} \delta},
$$

where $\alpha$ and $\beta$ stand for a set of quantum numbers $(j, \boldsymbol{k})$, $g_{\mathrm{s}}=2$ and $g_{\mathrm{v}}=2$ are the spin and valley degeneracy, $\delta$ is phenomenological broadening, and $f_{\alpha}=f\left(\varepsilon_{\alpha}\right)$ is the Fermi distribution function. We have used the fact that both $\mathrm{K}$ and $\mathrm{K}$ ' points give the same contribution. Explicitly, we have

$$
\begin{aligned}
\sigma_{z z}(\omega) & =\mathrm{i} \omega e^{2} d^{2} \frac{g_{\mathrm{v}} g_{\mathrm{s}}}{4} \frac{1}{2 \pi \gamma^{2}} \sum_{j, j^{\prime}} \int_{0}^{\infty} \gamma^{2} k \mathrm{~d} k g_{0}(\gamma k) \\
& \times \frac{\left[f\left(\varepsilon_{j k}\right)-f\left(\varepsilon_{j^{\prime} k}\right)\right]\left|\hat{\tau}_{j j^{\prime}}(k)\right|^{2}}{\varepsilon_{j k}-\varepsilon_{j^{\prime} k}+\hbar \omega+\mathrm{i} \delta}
\end{aligned}
$$

In the above, we have introduced cutoff function,

$$
g_{0}(\varepsilon)=\frac{\varepsilon_{\mathrm{c}}^{n_{\mathrm{c}}}}{\varepsilon_{\mathrm{c}}^{n_{\mathrm{c}}}+\varepsilon^{n_{\mathrm{c}}}},
$$

with cutoff energy $\varepsilon_{\mathrm{c}} \approx 3 \gamma_{0}$ corresponding to the half of the $\pi$-band width and $n_{\mathrm{c}}$ should be chosen in such a way that the integral should converge. The typical magnitude of the conductivity becomes

$$
\sigma_{0}=\frac{e^{2}}{\hbar} \frac{g_{\mathrm{v}} g_{\mathrm{s}}}{4} \frac{d^{2}}{2 \pi \gamma^{2}} \Delta^{2} \sim \frac{e^{2}}{\pi \hbar} \frac{g_{\mathrm{v}} g_{\mathrm{s}}}{4} \times 0.022,
$$

where we have used $a=2.46 \AA, d=3.34 \AA, \Delta \approx 0.4 \mathrm{eV}$, and $\gamma_{0} \approx 3 \mathrm{eV}$ in the last expression.

\subsection{Symmetric Bilayer}

In the symmetric case, in particular, we can make some analytic treatments. Let us define

$$
\begin{aligned}
\varepsilon(k) & =\sqrt{\left(\frac{\Delta}{2}\right)^{2}+(\gamma k)^{2}}, \\
\gamma k & =\varepsilon(k) \sin \psi, \\
\frac{\Delta}{2} & =\varepsilon(k) \cos \psi,
\end{aligned}
$$

where $\psi$ is 0 at $k=0$ and approaches $\pi / 2$ when $\gamma k \gg \Delta / 2$. Then, the eigenenergies are given by

$$
\begin{aligned}
& \varepsilon_{s 1}(k)=2 s \varepsilon(k) \sin ^{2}(\psi / 2), \\
& \varepsilon_{s 2}(k)=2 s \varepsilon(k) \cos ^{2}(\psi / 2),
\end{aligned}
$$

and the corresponding wave functions are given by

$$
\boldsymbol{F}_{\text {snk }}(\boldsymbol{r})=\frac{1}{L} \exp (\mathrm{i} \boldsymbol{k} \cdot \boldsymbol{r}) U[\theta(\boldsymbol{k})] \boldsymbol{F}_{\text {sn } \boldsymbol{k}},
$$

where $L^{2}$ is the area of the system,

$$
U(\theta)=\left(\begin{array}{cccc}
1 & 0 & 0 & 0 \\
0 & \mathrm{e}^{\mathrm{i} \theta} & 0 & 0 \\
0 & 0 & \mathrm{e}^{\mathrm{i} \theta} & 0 \\
0 & 0 & 0 & \mathrm{e}^{2 \mathrm{i} \theta}
\end{array}\right),
$$


and

$$
\begin{gathered}
\boldsymbol{F}_{s 1 k}=\frac{1}{\sqrt{2}}\left(\begin{array}{r}
s \cos (\psi / 2) \\
\sin (\psi / 2) \\
-s \sin (\psi / 2) \\
-\cos (\psi / 2)
\end{array}\right), \\
\boldsymbol{F}_{s 2 k}=\frac{1}{\sqrt{2}}\left(\begin{array}{r}
s \sin (\psi / 2) \\
\cos (\psi / 2) \\
s \cos (\psi / 2) \\
\sin (\psi / 2)
\end{array}\right) .
\end{gathered}
$$

We have the correspondence shown in Table I between quantum number $j$ and $(s, n)$ with $s= \pm$ and $n=1,2$. The matrix elements of $\hat{\tau}$ are calculated as in Table II.

Consider the case that the Fermi level lies in the band $j=3$ or $(+, 1)$. For the transition $(+, 1) \rightarrow(+, 2)$, in particular, we have

$$
\varepsilon_{+2}(k)-\varepsilon_{+1}(k)=\Delta,
$$

independent of $k$. Further, we have

$$
(+, 2|\hat{\tau}|+, 1)=\sin \psi .
$$

Define $n_{\mathrm{s}}^{0}$ be the electron concentration when the Fermi level reaches the bottom of band $(+, 2)$ or $\varepsilon_{F}=\Delta$, i.e.,

$$
n_{\mathrm{s}}^{0}=\frac{g_{\mathrm{v}} g_{\mathrm{s}}}{2 \pi \gamma^{2}} \Delta^{2} .
$$

Then, the transition strength averaged over states up to the Fermi energy becomes

$$
\left\langle\sin ^{2} \psi\right\rangle=1-\frac{1}{8} \frac{n_{\mathrm{s}}^{0}}{n_{\mathrm{s}}} \ln \left(1+8 \frac{n_{\mathrm{s}}}{n_{\mathrm{s}}^{0}}\right),
$$

where $\langle\cdots\rangle$ means the average over states with energy lying between $\varepsilon=0$ and $\varepsilon_{\mathrm{F}}$. Then, we have

$$
\tilde{\sigma}_{z z}(\omega)=\sigma_{0} \frac{\hbar \omega}{\Delta} \frac{n_{\mathrm{s}}}{n_{\mathrm{s}}^{0}} \frac{(-2 \mathrm{i})\left\langle\sin ^{2} \psi\right\rangle \Delta^{2}}{(1+\beta) \Delta^{2}-(\hbar \omega+\mathrm{i} 0)^{2}},
$$

with

$$
\beta=8 \pi \frac{g_{\mathrm{v}} g_{\mathrm{s}}}{4} \frac{e^{2}}{\kappa d} \frac{d^{2}}{2 \pi \gamma^{2}} \Delta\left\langle\sin ^{2} \psi\right\rangle \frac{n_{\mathrm{s}}}{n_{\mathrm{s}}^{0}} .
$$

This shows that the resonance energy is shifted from $\Delta$ to $\sqrt{1+\beta} \Delta$ due to the depolarization effect. For $n_{\mathrm{s}} / n_{\mathrm{s}}^{0}=1$, in particular, we have $\left\langle\sin ^{2} \psi\right\rangle=0.725 \cdots$ and $\beta=0.68 \cdots$, giving about $30 \%$ shift of the resonance energy to the higher energy side.

For the transition $(-, 1) \rightarrow(+, 1)$, we have $\mid(-, 1|\tau|$ $+, 1)\left.\right|^{2}=\cos ^{2} \psi \approx 1$ and

$$
\varepsilon_{+1}(k)-\varepsilon_{-1}(k) \approx \frac{\hbar^{2} k^{2}}{m^{*}},
$$

with

$$
\frac{\hbar^{2}}{2 m^{*}}=\frac{\gamma^{2}}{\Delta}
$$

Then, we have

$$
\begin{aligned}
\sigma_{z z}(\omega) \approx \sigma_{0} \frac{\hbar \omega}{4 \Delta} & \left(\pi \theta\left[(\hbar \omega)^{2}-\left(\frac{2 \gamma^{2} k_{F}^{2}}{\Delta}\right)^{2}\right]\right. \\
& \left.-\mathrm{i} \ln \left|\frac{4 \varepsilon_{c}^{4} / \Delta^{2}}{\left(2 \gamma^{2} k_{F}^{2} / \Delta\right)^{2}-(\hbar \omega)^{2}}\right|\right) .
\end{aligned}
$$

This shows that the absorption exhibits a step-function- like behavior with a spectral edge suppressed by the logarithmic divergence of $\varepsilon(\omega)$.

For the transition $(-, 2) \rightarrow(+, 2)$, we have

$$
\varepsilon_{+2}(k)-\varepsilon_{-2}(k) \approx 2 \Delta+\frac{\hbar^{2} k^{2}}{m^{*}} .
$$

Then, we have

$$
\sigma_{z z}(\omega) \approx \sigma_{0}\left(\frac{\pi}{2} \theta\left[(\hbar \omega)^{2}-(2 \Delta)^{2}\right]-\frac{\mathrm{i}}{2} \ln \left|\frac{2 \Delta+\hbar \omega}{2 \Delta-\hbar \omega}\right|\right),
$$

showing that the absorption exhibits a step-function-like behavior with a suppressed edge.

Transitions $(-, 1) \rightarrow(+, 2)$ and $(-, 2) \rightarrow(+, 1)$ are both forbidden in a symmetric bilayer. When slight asymmetry in the potential is introduced, however, bands $(-, 1)$ and $(+, 1)$ start to be mixed with each other. The effective Hamiltonian becomes

$$
\mathcal{H}_{\text {eff }}=\left(\begin{array}{cc}
(+, 1) & (-, 1) \\
+2 \varepsilon(k) \sin ^{2}(\psi / 2) & -(e F d / 2) \cos \psi \\
-(e F d / 2) \cos \psi & -2 \varepsilon(k) \sin ^{2}(\psi / 2)
\end{array}\right) .
$$

Thus, for $k=0$, two states are mixed with equal amplitude. As a result, the transitions from $(-, 1)$ to $(+, 2)$ and from $(-, 2)$ to $(+, 1)$ become allowed in the vicinity of $k=0$ and a small absorption appears near $\hbar \omega \approx \Delta+|e F d|$ for nonzero $F$ (see $\S 4$ ).

\subsection{Optical Absorption for Parallel Polarization}

The dynamical conductivity describing optical absorption for electric field parallel to the layer was calculated previously, ${ }^{30)}$ and therefore we shall give only the final results. The dynamical conductivity becomes

$$
\sigma_{x x}(\omega)=\frac{g_{\mathrm{v}} g_{\mathrm{s}}}{L^{2}}\left(\frac{e \gamma}{\hbar}\right)^{2} \frac{\hbar}{\mathrm{i}} \sum_{\alpha, \beta} \frac{\left|\left(\alpha\left|\Sigma_{x}\right| \beta\right)\right|^{2}\left(f_{\alpha}-f_{\beta}\right)}{\left(\varepsilon_{\alpha}-\varepsilon_{\beta}\right)\left(\varepsilon_{\alpha}-\varepsilon_{\beta}+\hbar \omega+\mathrm{i} \delta\right)},
$$

with

$$
\Sigma_{x}=\left(\begin{array}{llll}
0 & 1 & 0 & 0 \\
1 & 0 & 0 & 0 \\
0 & 0 & 0 & 1 \\
0 & 0 & 1 & 0
\end{array}\right) .
$$

This can be separated into the Drude (intra-band) part and the inter-band part

$$
\begin{gathered}
\sigma_{x x}(\omega)=\frac{g_{\mathrm{v}} g_{\mathrm{s}}}{L^{2}}\left(\frac{e \gamma}{\hbar}\right)^{2} \frac{\hbar}{\mathrm{i}}\left[\sum_{j \boldsymbol{k}} \frac{\partial f\left(\varepsilon_{j k}\right)}{\partial \varepsilon_{j k}} \frac{\left|\left(j \boldsymbol{k}\left|\Sigma_{x}\right| j \boldsymbol{k}\right)\right|^{2}}{\hbar \omega+\mathrm{i} \delta}\right. \\
\left.-\sum_{j \neq j^{\prime}, \boldsymbol{k}} \frac{\left|\left(j \boldsymbol{k}\left|\Sigma_{x}\right| j^{\prime} \boldsymbol{k}\right)\right|^{2}\left[f\left(\varepsilon_{j k}\right)-f\left(\varepsilon_{j^{\prime} k}\right)\right](\hbar \omega+\mathrm{i} \delta)}{\left(\varepsilon_{j k}-\varepsilon_{j^{\prime} k}\right)\left[\left(\varepsilon_{j k}-\varepsilon_{j^{\prime} k}\right)^{2}-(\hbar \omega+\mathrm{i} \delta)^{2}\right]}\right] \cdot(2
\end{gathered}
$$

The squared matrix element for a symmetric bilayer is listed in Table III. At the band edges $k=0$ or $\psi=0$, the selection rule for the parallel and perpendicular polarized light is completely different. The dynamical conductivity for $\varepsilon_{\mathrm{F}}=0$ has essentially no prominent structure except a step-like increase corresponding to transitions from $(-, 1)$ to $(+, 2){ }^{30)}$ With increase in $\varepsilon_{\mathrm{F}}$, a delta-function 
peak appears at $\hbar \omega=\Delta$ with intensity proportional to $\left|\varepsilon_{\mathrm{F}}\right|$, corresponding to allowed transitions $(+, 1) \rightarrow(+, 2)$.

\section{$\S 3$. Numerical Results}

In the present system we have electron-hole symmetry in the energy bands. As a result the dynamical conductivity describing optical properties has symmetry

$$
\sigma\left(\omega ;-n_{\mathrm{s}},-e F_{\mathrm{ext}}\right)=\sigma\left(\omega ;+n_{\mathrm{s}},+e F_{\mathrm{ext}}\right),
$$

and therefore we shall exclusively consider the case $n_{\mathrm{s}} \geq$ 0 . The results for $n_{\mathrm{s}}<0$ can be obtained by using the above relation. Figure 3 shows some examples of calculated dynamical conductivity for (a) perpendicular and (b) parallel polarization in a symmetric case $e F d=0$, with several values of the Fermi energy. The dashed lines in (a) represent $\sigma_{z z}(\omega)$ without depolarization effect and the solid lines $\tilde{\sigma}_{z z}(\omega)$ with depolarization effect. We assume broadening parameter $\delta / \Delta=0.02$.

One most prominent feature is the appearance of a sharp peak corresponding to transitions from $(+, 1)$ to $(+, 2)$ with the increase of $\varepsilon_{\mathrm{F}}$. In the clean limit $\delta \rightarrow 0$, the peak becomes a $\delta$ function because the bands are parallel to each other. For perpendicular polarization, this sharp peak is shifted to the higher energy side due to the depolarization effect. When the Fermi level reaches the bottom of band $(+, 2)$, this shift is as large as $30 \%$ as has been estimated in the previous section.

Figure 4 shows the dynamical conductivity for varying electron concentration $n_{\mathrm{s}}$ for $F_{\text {ext }}=0$, i.e., when the bilayer is symmetric for $n_{\mathrm{s}}=0$ and the asymmetry potential $e F d$ increases with the change of $n_{\mathrm{s}}$. The energies where interband transitions start to appear are shown by thin dotted lines. For transitions from $(+, 1)$ to $(+, 2)$, i.e., $3 \rightarrow 4$, the upper edge where the transition disappears is also included.

For the parallel polarization, the absorption corresponding to transitions $(+, 1) \rightarrow(+, 2)$ becomes broadened with increase of $n_{\mathrm{s}}$ due to the gap opening. For the perpendicular polarization, on the other hand, this broadening is not significant and exhibits a similar amount of shift due to the depolarization effect. This is because the resonance, i.e., the zero point of $\varepsilon(\omega)$, occurs at an energy higher than the interband continuum due to the depolarization effect. A weak structure appears near the line denoted as $2 \rightarrow 4$ with the increase of $n_{\mathrm{s}}$. This corresponds to the transition from $(-, 1)$ to $(+, 2)$, which becomes allowed by the band-gap opening as mentioned in the end of the last section.

Similar results for $e F_{\text {ext }} d / \Delta=1$ are shown in Fig. 5. For the parallel polarization, the absorption corresponding to transitions $(+, 1) \rightarrow(+, 2)$ is considerably broadened due to further increase of the gap with $n_{\mathrm{s}}$. The absorption for the perpendicular polarization tends to have a sharper peak near the higher-energy edge of the interband continuum and exhibits a sharp peak for sufficiently large $n_{\mathrm{s}}$ where the zero of $\varepsilon(\omega)$ is well separated from the continuum. The transition from $(-, 1)$ to $(+, 2)$ denoted as $2 \rightarrow 4$ is now much more prominent. The peak appearing at $\hbar \omega / \Delta \sim 1$ at $n_{\mathrm{s}}=0$ for the parallel polarization is due to the opening of gap $\sim|e F d|$.

Figure 6 shows results for $e F_{\text {ext }} d / \Delta=-1$ corresponding to asymmetry opposite to Fig. 5 . In this case the asymmetry becomes weaker with $n_{\mathrm{s}}$ and therefore the peak corresponding to transitions $(+, 1) \rightarrow(+, 2)$ becomes sharper with $n_{\mathrm{s}}$. Further, the transition from $(-, 1)$ to $(+, 2)$ denoted as $2 \rightarrow 4$ for the perpendicular polarization becomes weaker with the increase of $n_{\mathrm{s}}$.

\section{$\S 4$. Discussion and Summary}

We have calculated the optical absorption spectrum for light with electric field perpendicular to bilayer graphene and shown that the spectrum is quite different from that for the parallel polarization. This arises partly from the difference in the selection rule and from the important depolarization effect due to induced charge imbalance between two layers. The depolarization effect is most prominent for the sharp transition $(+, 1) \rightarrow(+, 2)$ almost parallel to each other.

The strength of the absorption for the perpendicular polarization is determined by conductivity $\tilde{\sigma}_{z z}(\omega)$ of the order of $\sigma_{0}$, while that for the parallel polarization by $\sigma_{x x}(\omega)$ of the order of $\left(g_{\mathrm{v}} g_{\mathrm{s}} e^{2}\right) /(4 \pi \hbar)$. Because $\sigma_{0} \sim 0.022 \times\left(g_{\mathrm{v}} g_{\mathrm{s}} e^{2}\right) /(4 \pi \hbar)$, the absorption intensity for perpendicular polarization is much smaller. As a result, for light incident with certain angle from the vertical direction the absorption by the field component perpendicular to the layer is small and usually negligible.

This smallness of $\tilde{\sigma}_{z z}(\omega)$ mainly comes from the fact that inter-layer coupling $\gamma_{1}(\sim 0.4 \mathrm{eV})$ is much smaller than intra-layer coupling $\gamma_{0}(\sim 3 \mathrm{eV})$ in spite of the fact that inter-layer separation $d(=3.34 \AA)$ is not so much different from the intralayer lattice constant $a(=2.46$ $\AA)$. This is quite in contrast to conventional space-charge layers on semiconductor surfaces where $\tilde{\sigma}_{z z}(\omega)$ is not so much different from $\sigma_{x x}(\omega) .{ }^{19)}$ Usually, $\tilde{\sigma}_{z z}(\omega)$ can be experimentally measured using the configuration of a transmission line. ${ }^{4344)}$ The weak absorption intensity discussed above requires the use of bilayer graphene with large area.

\section{Acknowledgments}

This work was supported in part by Grant-in-Aid for Scientific Research on Priority Area "Carbon Nanotube Nanoelectronics," by Grant-in-Aid for Scientific Research, and by Global Center of Excellence Program at Tokyo Tech "Nanoscience and Quantum Physics" from Ministry of Education, Culture, Sports, Science and Technology Japan.

\section{References}

1) K. S. Novoselov, A. K. Geim, S. V. Morozov, D. Jiang, M. I. Katsnelson, I. V. Grigorieva, S. V. Dubonos, and A. A. Firsov, Nature 438 (2005) 197.

2) K. S. Novoselov, E. McCann, S. V. Morozov, V. I. Falko, M. I. Katsnelson, U. Zeitler, D. Jiang, F. Schedin, and A. K. Geim, Nature Phys. 2 (2006) 177.

3) T. Ohta, A. Bostwick, T. Seyller, K. Horn, and E. Rotenberg, Science 313 (2006) 951

4) E. V. Castro, K. S. Novoselov, S. V. Morozov, N. M. R. Peres, J. M. B. Lopes dos Santos, J. Nilsson, F. 
Guinea, A. K. Geim, and A. H. Castro Neto, Phys. Rev. Lett. 99 (2007) 216802.

5) J. B. Oostinga, H. B. Heersche, X.-L. Liu, A. F. Morpurgo, and L. M. K. Vandersypen, Nat. Mat. 7 (2008) 151.

6) E. A. Henriksen, Z. Jiang, L.-C. Tung, M. E. Schwartz, M. Takita, Y.-J. Wang, P. Kim, and H. L. Stormer, Phys. Rev. Lett. 100 (2008) 087403.

7) J. W. McClure, Phys. Rev. 104 (1956) 666.

8) J. C. Slonczewski and P. R. Weiss, Phys. Rev. 109 (1958) 272.

9) D. P. DiVincenzo and E. J. Mele, Phys. Rev. B 29 (1984) 1685.

10) G. W. Semenoff, Phys. Rev. Lett. 53 (1984) 2449.

11) H. Ajiki and T. Ando, J. Phys. Soc. Jpn. 62 (1993) 1255 .

12) C. L. Kane and E. J. Mele, Phys. Rev. Lett. 78 (1997) 1932.

13) T. Ando, J. Phys. Soc. Jpn. 74 (2005) 777 and references cited therein.

14) T. Ando, Physica E 40 (2007) 213.

15) N. H. Shon and T. Ando, J. Phys. Soc. Jpn. 67 (1998) 2421

16) Y. Zheng and T. Ando, Phys. Rev. B 65 (2002) 245420 .

17) T. Ando, Y. Zheng, and H. Suzuura, J. Phys. Soc. Jpn. 71 (2002) 1318.

18) H. Suzuura and T. Ando, Phys. Rev. Lett. 89 (2002) 266603.

19) T. Ando, A. B. Fowler, and F. Stern, Rev. Mod. Phys. 54 (1982) 437 and references cited therein.

20) E. McCann and V. I. Falko, Phys. Rev. Lett. 96 (2006) 086805.

21) F. Guinea, A. H. Castro Neto, and N. M. R. Peres, Phys. Rev. B 73 (2006) 245426.

22) S. Latil and L. Henrard, Phys. Rev. Lett. 97 (2006) 036803.

23 ) B. Partoens and F. M. Peeters, Phys. Rev. B 74 (2006) 075404.

24) C. L. Lu, C. P. Chang, Y. C. Huang, J. M. Lu, C. C. Hwang, and M. F. Lin, J. Phys.: Cond. Matt. 18 (2006) 5849.

25) M. Koshino and T. Ando, Phys. Rev. B 73 (2006) 245403.

26) M. Koshino and T. Ando, AIP Conf. Proc. 893 (2007) 621.

27) J. Nilsson, A. H. Castro Neto, F. Guinea, and N. M. R. Peres, Phys. Rev. Lett. 97 (2006) 266801.

28) J. Nilsson and A. H. Castro Neto, Phys. Rev. Lett. 98 (2007) 126801.

29) S. Adam and S. Das Sarma, Phys. Rev. B 77 (2008) 115436.

$30)$ D. S. L. Abergel and V. I. Falko, Phys. Rev. B 75 (2007) 155430.

31) C. L. Lu, C. P. Chang, Y. C. Huang, R. B. Chen, and M. L. Lin, Phys. Rev. B 73 (2006) 144427.

32) M. Koshino and T. Ando, Phys. Rev. B 77 (2008) 115313.

33) M. Koshino and T. Ando, Phys. Rev. B 76 (2007) 085425 .

34) M. Nakamura and L. Hirasawa, Phys. Rev. B 77 (2008) 045429.

35) E. McCann, Phys. Rev. B 74 (2006) 161403.

36) H. Min, B. Sahu, S. K. Banerjee, and A. H. MacDonald, Phys. Rev. B 75 (2007) 155115.

37) T. Ando and M. Koshino, J. Phys. Soc. Jpn. 78
(2009) 034709.

38) P. R. Wallace, Phys. Rev. 71 (1947) 622.

39) G. S. Painter and D. E. Ellis, Phys. Rev. B 1 (1970) 4747.

40) W. W. Toy, M. S. Dresselhaus, and G. Dresselhaus, Phys. Rev. B 15 (1977) 4077.

41) A. Misu, E. Mendez, and M. S. Dresselhaus, J. Phys. Soc. Jpn. 47 (1979) 199.

42) T. Ando, J. Phys. Soc. Jpn. 76 (2007) 024712.

43) A. Kamgar, P. Kneschaurek, G. Dorda, and J. F. Koch, Phys. Rev. Lett. 32 (1974) 1251.

44) P. Kneschaurek, A. Kamgar, and J. F. Koch, Phys. Rev. B 14 (1976) 1610.

Table I The correspondence between level index $j$ and $(s, n)$.

\begin{tabular}{ccccc}
\hline \hline$j$ & 1 & 2 & 3 & 4 \\
\hline$(s, n)$ & $(-, 2)$ & $(-, 1)$ & $(+, 1)$ & $(+, 2)$ \\
\hline
\end{tabular}

Table II Calculated matrix elements of operator $\hat{\tau}$.

\begin{tabular}{ccccc}
\hline \hline$\hat{\tau}$ & $4(+, 2)$ & $3(+, 1)$ & $2(-, 1)$ & $1(-, 2)$ \\
\hline $4(+, 2)$ & 0 & $+\sin \psi$ & 0 & $+\cos \psi$ \\
\hline $3(+, 1)$ & $+\sin \psi$ & 0 & $-\cos \psi$ & 0 \\
\hline $2(-, 1)$ & 0 & $-\cos \psi$ & 0 & $+\sin \psi$ \\
\hline $1(-, 2)$ & $+\cos \psi$ & 0 & $+\sin \psi$ & 0 \\
\hline
\end{tabular}

Table III Calculated current matrix elements $\mid\left(j \boldsymbol{k}\left|\Sigma_{x}\right|\right.$ $\left.j^{\prime} \boldsymbol{k}\right)\left.\right|^{2}$ averaged over the direction of the wave vector.

\begin{tabular}{ccccc}
\hline \hline$\left\langle\left|\left(j\left|\Sigma_{x}\right| j^{\prime}\right)\right|^{2}\right\rangle$ & $(+, 2)$ & $(+, 1)$ & $(-, 1)$ & $(-, 2)$ \\
\hline$(+, 2)$ & $\frac{1}{2} \sin ^{2} \psi$ & $\frac{1}{2} \cos ^{2} \psi$ & $\frac{1}{2} \cos ^{2} \psi$ & $\frac{1}{2} \sin ^{2} \psi$ \\
\hline$(+, 1)$ & $\frac{1}{2} \cos ^{2} \psi$ & $\frac{1}{2} \sin ^{2} \psi$ & $\frac{1}{2} \sin ^{2} \psi$ & $\frac{1}{2} \cos ^{2} \psi$ \\
\hline$(-, 1)$ & $\frac{1}{2} \cos ^{2} \psi$ & $\frac{1}{2} \sin ^{2} \psi$ & $\frac{1}{2} \sin ^{2} \psi$ & $\frac{1}{2} \cos ^{2} \psi$ \\
\hline$(-, 2)$ & $\frac{1}{2} \sin ^{2} \psi$ & $\frac{1}{2} \cos ^{2} \psi$ & $\frac{1}{2} \cos ^{2} \psi$ & $\frac{1}{2} \sin ^{2} \psi$ \\
\hline
\end{tabular}

\section{Figure Captions}

Fig. 1 (a) The coordinates for a bilayer graphene lying parallel to the $x y$ plane. The top and bottom layers are at $z=-d / 2$ and $+d / 2$, respectively. (b) The potential distribution associated with polarization between two layers.

Fig. 2 (Color online) The energy dispersion for varying values of the potential difference $e F d$. The dot-dotdashed lines show that of a monolayer graphene. The parameter $\Delta=\gamma_{1}$ represents the nearestneighbor interlayer coupling.

Fig. 3 (Color online) Calculated dynamical conductivity for (a) perpendicular and (b) parallel polarization in a symmetric bilayer graphene. The dashed lines in (a) represent $\sigma_{z z}(\omega)$ without depolarization effect and the solid lines $\tilde{\sigma}_{x x}(\omega)$ with depolarization effect.

Fig. 4 (Color online) Calculated dynamical conductivity for (a) perpendicular and (b) parallel polarization for $e F_{\text {ext }} d=0$. The potential difference between two layers, $e F d / \Delta$, self-consistently determined is 
listed in the figure. Thin dotted lines denote edges of interband transitions $\left(j \rightarrow j^{\prime}\right.$ means those from band $j$ to band $j^{\prime}$ ).

Fig. 5 (Color online) Calculated dynamical conductivity for (a) perpendicular and (b) parallel polariza- tion for $e F_{\text {ext }} d / \Delta=1$.

Fig. 6 (Color online) Calculated dynamical conductivity for (a) perpendicular and (b) parallel polarization for $e F_{\text {ext }} d / \Delta=-1$. 
(a)

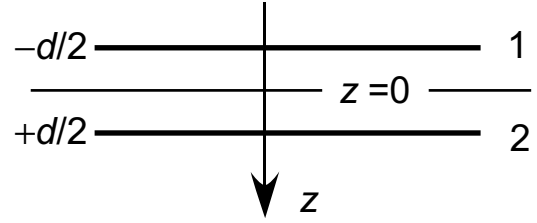

(b)

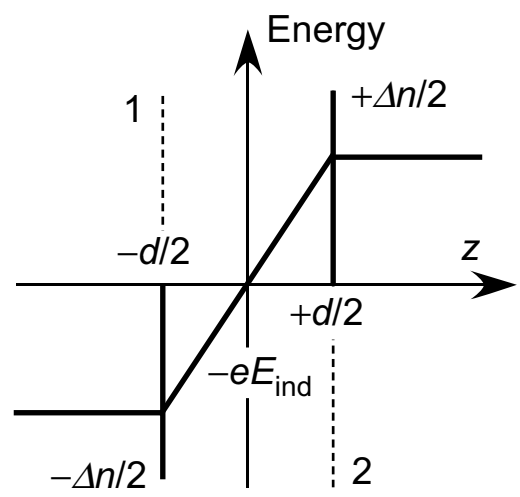

Fig. 1

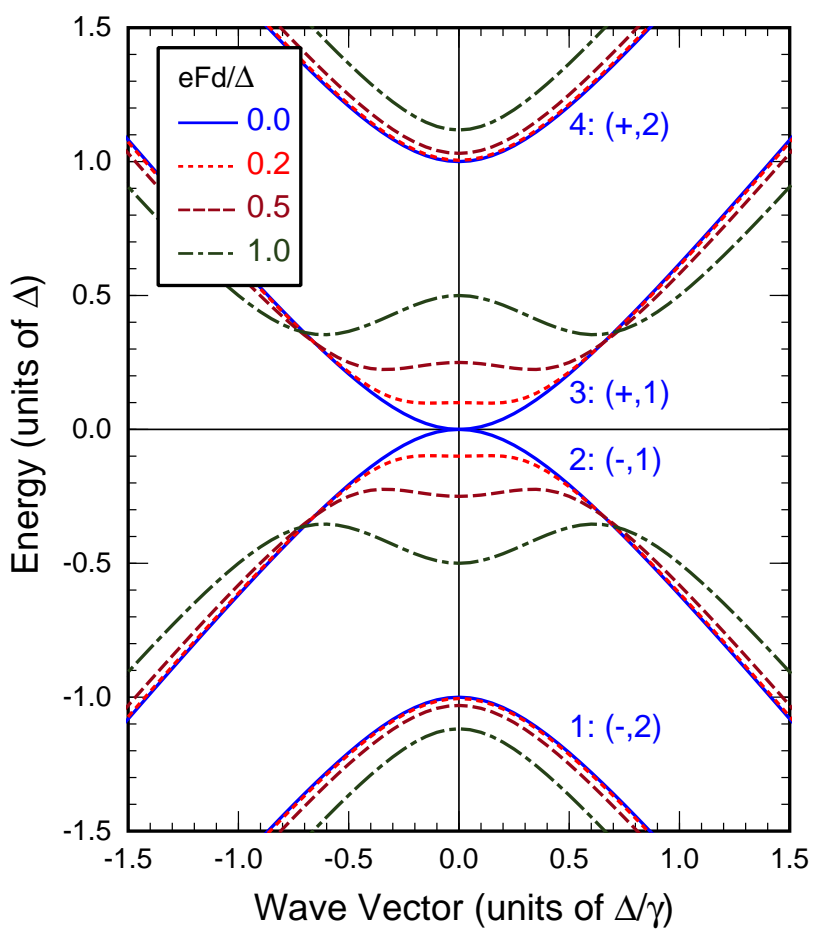

Fig. 2

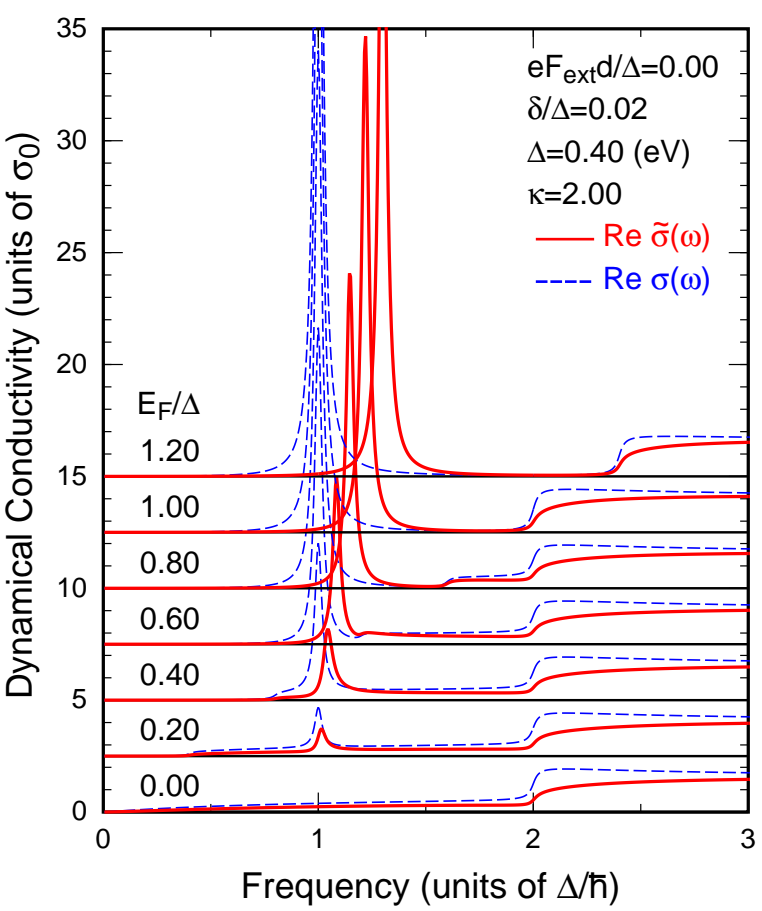

Fig. 3 (a)

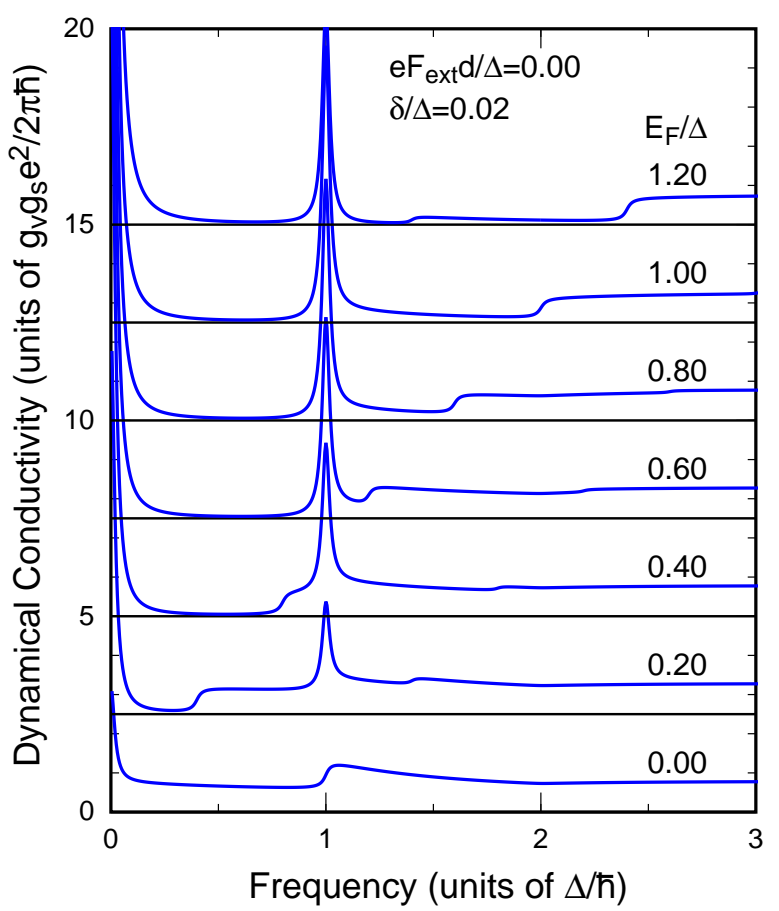

Fig. 3 (b) 


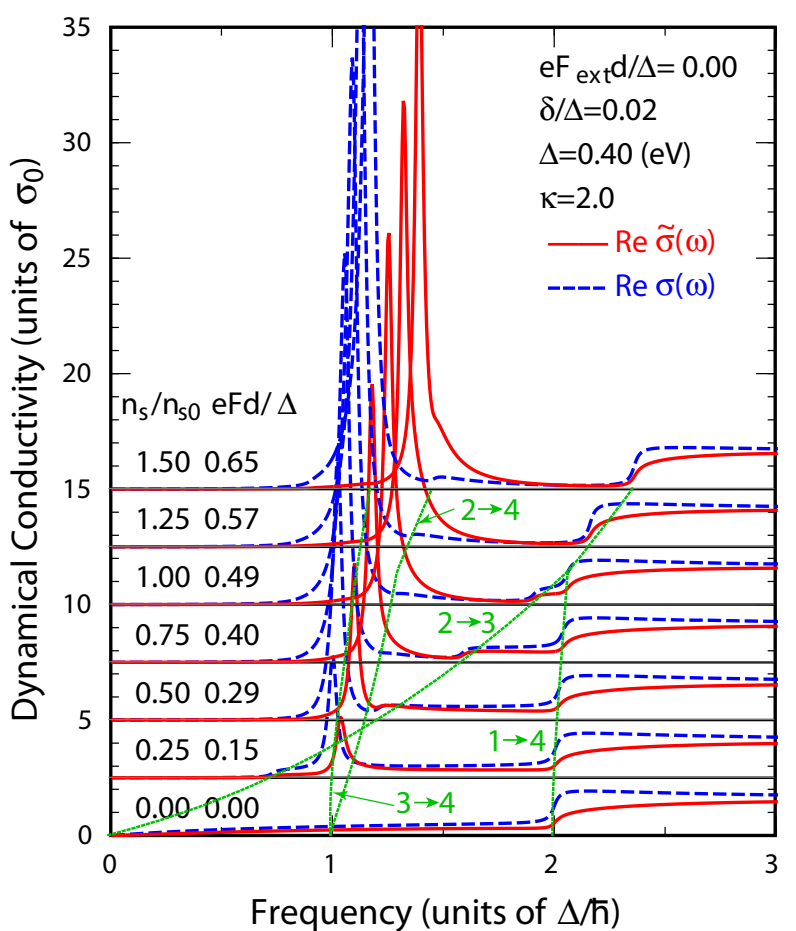

Fig. 4 (a)

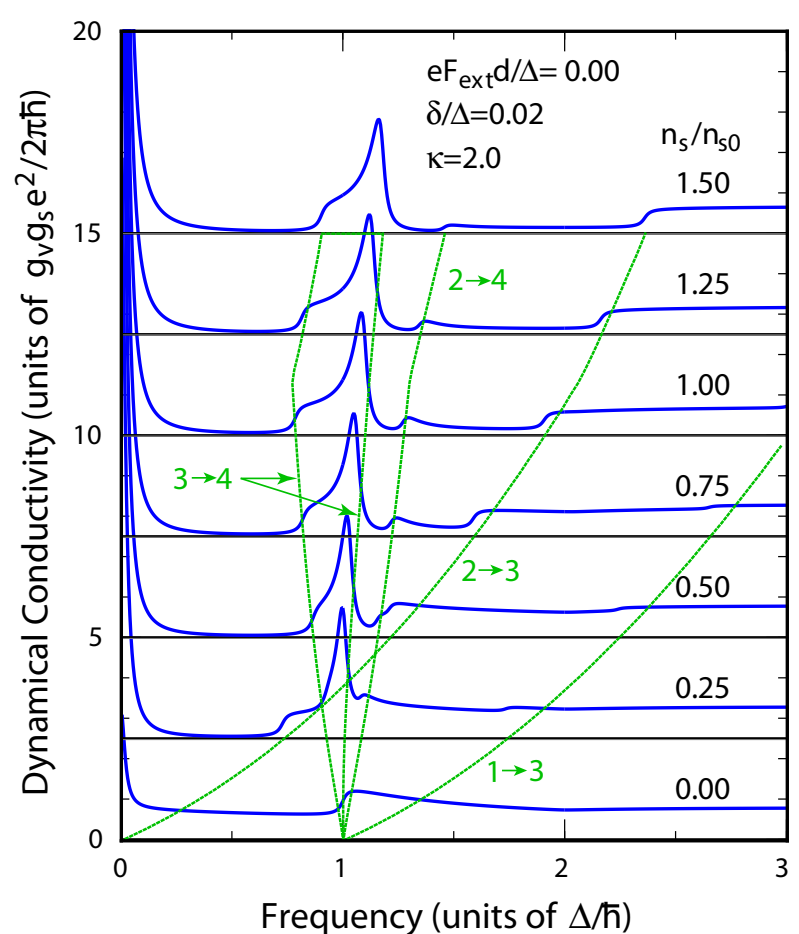

Fig. 4 (b)

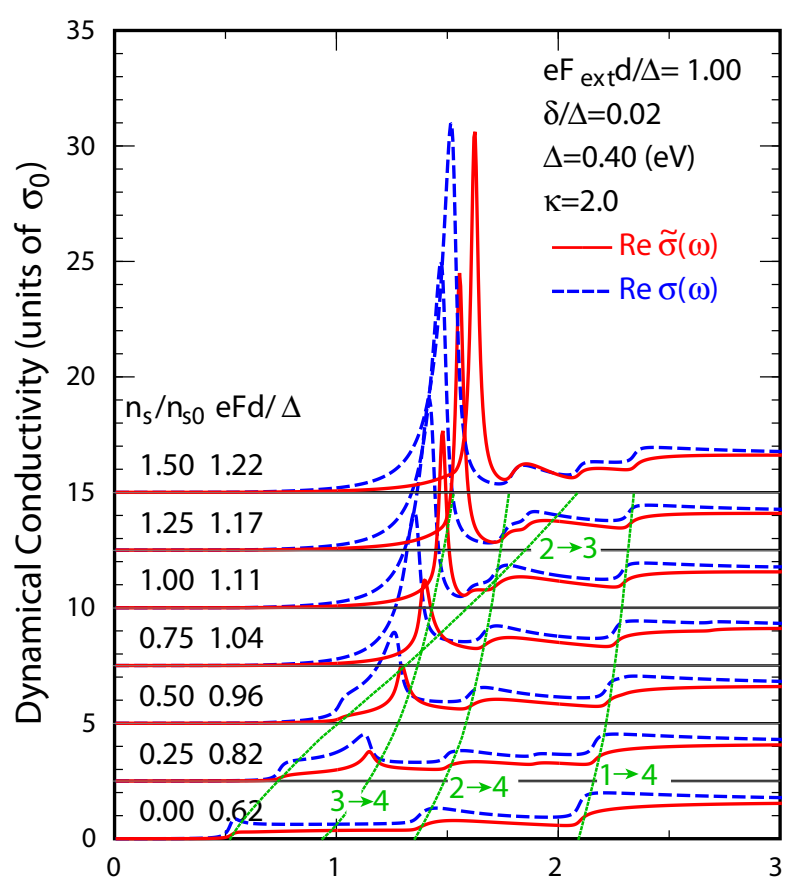

Frequency (units of $\Delta / \hbar$ )

Fig. 5 (a)

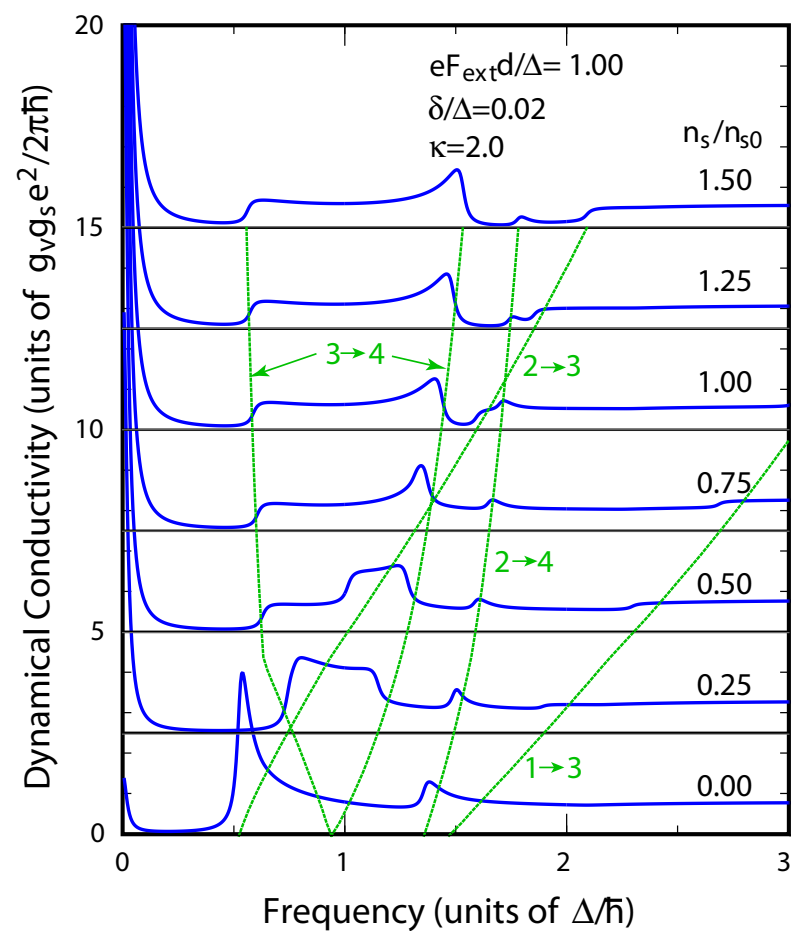

Fig. 5 (b) 


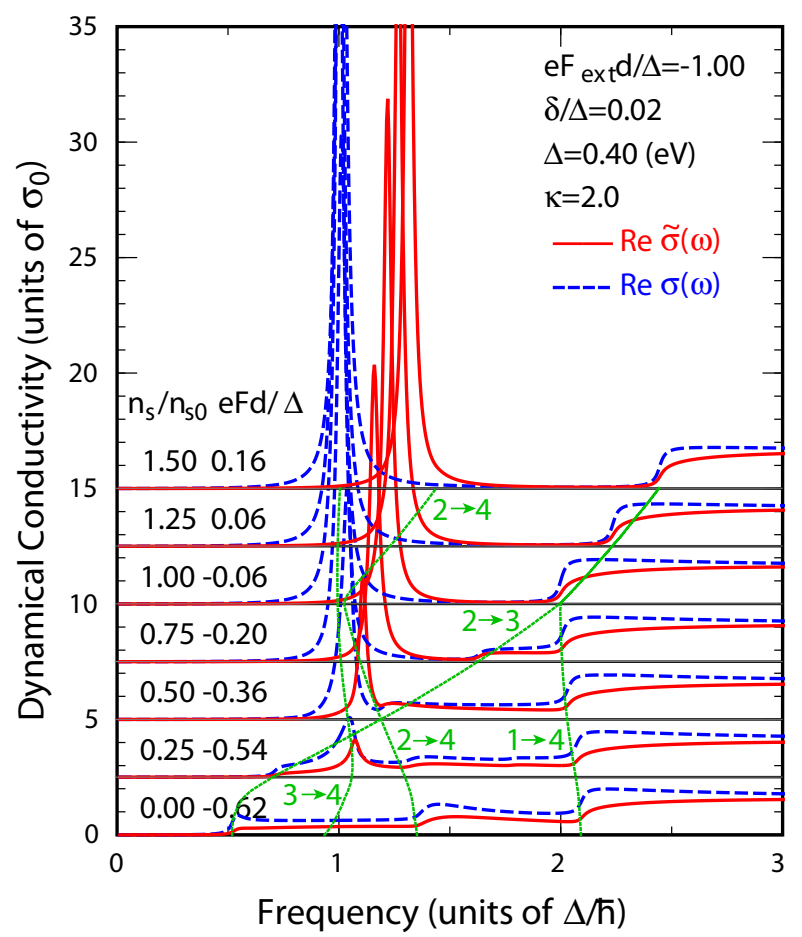

Fig. 6 (a)

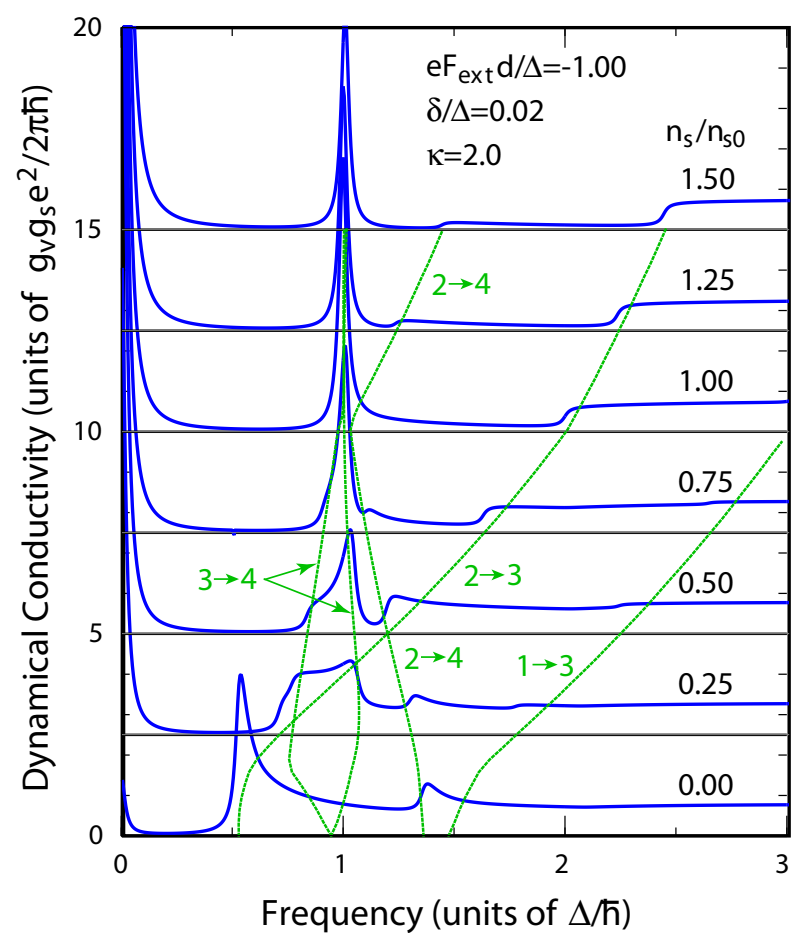

Fig. 6 (b) 